\title{
MOLECULAR AND STRUCTURAL BASIS OF THE EVOLUTION OF PARVOVIRUS TROPISM
}

\author{
P. TIJSSEN ${ }^{*}$ \\ Laboratory of Structural and Molecular Virology, Centre de Microbiologie et \\ Biotechnologie, INRS-Institut Armand-Frappier, Building 12, Université du Québec, \\ 531, boul. des Prairies, Laval, QC, Canada H7N 4 Z3
}

(Received March 12, 1999; accepted April 8, 1999)

\begin{abstract}
Parvoviruses have small genomes and, consequently, are highly dependent on their host for various functions in their reproduction. Since these viruses generally use ubiquitous receptors, restrictions are usually intracellularly regulated. A lack of mitosis, and hence absence of enzymes required for DNA replication, is a powerful block of virus infection. Allotropic determinants have been identified for several parvoviruses: porcine parvovirus, canine parvovirus (CPV), feline parvovirus (feline panleukopenia virus), minute virus of mice, Aleutian disease virus, and $G m \mathrm{DNV}$ (an insect parvovirus). Invariably, these identifications involved the use of infectious clones of these viruses and the exchange of restriction fragments to create chimeric viruses, of which the resulting phenotype was then established by transfection in appropriate cell lines. The tropism of these viruses was found to be governed by minimal changes in the sequence of the capsid proteins and, often, only 2 or 3 critical amino acids are responsible for a given tropism. These amino acids are usually located on the outside of the capsid near or on the spike of the threefold axis for the vertebrate parvoviruses and on loops 2 or 3 for the insect parvoviruses. This tropism is not mediated via specific cellular receptors but by interactions with intracellular factors. The nature of these factors is unknown but most data point to a stage beyond the conversion of the single-stranded DNA genome by host cell DNA polymerase into monomeric duplex intermediates of the replicative form. The sudden and devastating emergence of mink enteritis virus (MEV) and CPV in the last 50 years, and the possibility of more future outbreaks, demonstrates the importance of understanding parvovirus tropism.
\end{abstract}

Key words: Parvovirus, virus structure, virus tropism, viral evolution, host range

Parvoviruses, among the smallest viruses known, have a relatively simple, isometric capsid with a diameter of about $25 \mathrm{~nm}$ and contain a linear, singlestranded DNA genome of about 4-6 kb (Siegl, 1976; Tijssen, 1990; Berns, 1996; Tijssen et al., 1999). Some parvoviruses are autonomous, whereas others require

*E-mail: peter_tijssen@iaf.uquebec.ca; Fax: 1 (450) 6865626 
a helper virus (usually an adenovirus or a herpesvirus) for their replication. The limited coding capacity of the parvovirus genome implies a heavy dependence on cellular functions, especially those required for DNA replication and supplied by cells during the S-phase. As a result, autonomous parvoviruses infect dividing cells [e.g., in mitosis-active tissues, fetuses or tumour cells ('oncolytic')]. Pathogenesis is highly tissue specific, at least for the vertebrate parvoviruses, and productive parvoviral infections lead to cell lysis. Targeting of dividing cells, either 'mitotropism' or 'oncotropism', is therefore a sine qua non as the first level of tropism of parvoviruses.

The tropism of parvoviruses that require a helper virus, the adenoassociated viruses or AAVs, seems to be determined primarily by the tropism of their helper viruses. This 'virotropism' is usually much wider than tropism of autonomous parvoviruses. When AAV viruses enter a cell and are uncoated in the absence of a helper virus, their genomes can be integrated in a specific chromosome site to establish a latent infection (Kotin et al., 1992). These latent genomes can be activated by a subsequent helper virus infection. As a consequence, AAVs prevent superinfecting adenoviruses from transforming cells (Berns, 1996). Epidemiological studies have shown that, while $90 \%$ of the general population is seropositive for AAVs, patients suffering from cervical carcinoma are much less AAV-seropositive (Mayor et al., 1976). AAVs, in contrast to autonomous parvoviruses, have as yet to be associated with any pathogenicity and have received a considerable interest for potential use as vectors in gene therapy. Since goose and muscovy duck parvoviruses are closely related to these viruses (Zádori et al., 1995), it will be interesting to establish whether they are integration-competent and whether goose and duck adenoviruses could rescue these parvoviruses from non-susceptible cells.

Not all replicating cells are sensitive to autonomous parvoviruses and there are relatively few cell cultures that support parvoviruses. Cellular restrictions may act at different levels: (i) receptors; (ii) intracellular transport and decapsidation; (iii) virus-replication; and (iv) encapsidation and cell lysis. Allotropic determinants have been identified in the capsids of several animal and insect parvoviruses and often consist of just a few amino acids, but their cellular partners, that would interact with them, are hitherto elusive. In principle, these allotropic determinants could act at any of the four stages mentioned above but, as will be discussed in this review, most data point to the second and third stage.

Here, the biology and molecular biology of parvoviruses will be briefly reviewed, followed by a discussion of their three-dimensional structure and structure-function relationships, and finally the nature and evolution of viral tropism. 


\section{General properties and classification of parvoviruses}

Parvoviruses have been isolated from tissues from both vertebrates and invertebrates. Although other DNA virus families exist with members that infect either vertebrates or invertebrates (i.e., pox and iridoviruses), these replicate in the cytoplasm and contain considerable genetic information to code for proteins required for viral replication and, consequently, depend considerably less on host functions than parvoviruses. The observations that parvoviruses replicate in the nucleus, closely in tune with the physiology of the host cell, and are able to do so with both vertebrate and invertebrate cells, suggest that vertebrate and invertebrate parvoviruses may be more different than usually accepted. Indeed, when recently the first 3D structure of an invertebrate parvovirus was solved by X-ray crystallography (Simpson et al., 1998), major differences were observed between the two virus groups. Despite many differences in the molecular biology of these virus groups, it is surprising how many aspects of their genome organization and molecular biology are shared, justifying their classification as one family. For example, we discovered recently (Zádori et al., unpublished results) that the capsids of both vertebrate and invertebrate parvoviruses carry an enzymatic site with multiple activities (phospholipase A2 involved in infection process and signal transduction pathway). Similar activities have not yet been shown in other DNA viruses. The parvovirus family (Parvoviridae) is thus divided into two subfamilies, the Parvovirinae (vertebrates, i.e. human and other mammals) and the Densovirinae (invertebrates, i.e. insects, shrimps, crab, etc.).

The most recent classification of parvoviruses (Berns et al., 1995) needs to be revised. Classifications tend to evolve with the shift of research emphasis; for example, in early days, viruses were classified according to the symptoms of the diseases they caused (respiratory, hepatitis, etc.). Later, in the heyday of the biochemical and physicochemical characterization of viruses in the mid-sixties, Lwoff (1967) and Gibbs and Harrison (1968) each suggested a classification system based on these physicochemical properties. Currently, the classification hierarchy is governed by sequence homologies and molecular-biology functions and strategies. Conveniently, this did not change the general outline of the universal taxonomic system that evolved in the 1970s but merely fine-tuned it, particularly for the DNA viruses.

Although unofficial, the classification presented in Table 1 reflects, to our knowledge, best the hierarchy according to clades of similar sequences among parvoviruses. Compared to the official (ICTV) classification, many parvoviruses have been moved to other genera, some have been assigned a separate genus and, where necessary, names have been corrected. A comprehensive review of this classification is in progress (Zádori et al., unpublished results). Due to its unofficial nature, no attempt has been made at establishing a nomenclature for the genera. 


\section{Table 1}

Classification of parvoviruses according to sequence similarities (clades)

\begin{tabular}{|c|c|c|c|}
\hline Subfamily & Clade & (Genus) & Viruses and distinguishing features \\
\hline \multirow[t]{5}{*}{ Parvovirinae } & 1 & (Parvovirus) & $\begin{array}{l}\text { Porcine parvovirus (PPV), canine parvovirus, feline } \\
\text { parvovirus (= feline panleukopenia virus; FPV), mink } \\
\text { enteritis virus, raccoon parvovirus, minute virus of } \\
\text { mice, murine parvovirus, LuIII, Kilham rat virus, H-1. } \\
\text { Genome } 5 \mathrm{~kb} \text {, unique terminal hairpins, similar splicing } \\
\text { mechanisms; similar patterns of VPs }\end{array}$ \\
\hline & 2 & & $\begin{array}{l}\text { Aleutian Disease virus. Different genome organization } \\
\text { and VP pattern }\end{array}$ \\
\hline & 3 & & Bovine parvovirus. Genome $5.5 \mathrm{~kb}$ \\
\hline & 4 & (Erythrovirus) & $\begin{array}{l}\text { B19, simian parvovirus, chipmunk parvovirus. } \\
\text { Genome } 5.6 \mathrm{~kb} \text {, ITRs, One promoter }\end{array}$ \\
\hline & 5 & (Dependovirus) & $\begin{array}{l}\text { AAV (many serotypes), goose parvovirus, muscovy duck } \\
\text { parvovirus. Genome } 4.7 \mathrm{~kb} \text {. The genome termini of the } \\
\text { goose and duck parvoviruses resemble those of B } 19\end{array}$ \\
\hline \multirow[t]{6}{*}{ Densovirinae } & $1-\mathrm{a}$ & (Densovirus) & $\begin{array}{l}\text { Densoviruses from Galleria mellonella }(\text { GmDNV), } \\
\text { Pseudoplusia includens (PiDNV), Diatraea saccharalis } \\
\text { (DsDNV), Junonia coenia (JcDNV), Mythimna loreyi } \\
\text { (MlDNV). Ambisense genome of } 6 \mathrm{~kb} \text { (one strand } \\
\text { codes for NS proteins, one strand codes for VP pro- } \\
\text { teins), no splicing; large ITRs }\end{array}$ \\
\hline & $1-b$ & (Densovirus) & $\begin{array}{l}\text { Densovirus from Culex pipiens }(C p \mathrm{DNV}) ; 5.5 \mathrm{~kb} \\
\text { genome, ambisense; splicing in NS proteins }\end{array}$ \\
\hline & $1-c$ & (Densovirus) & $\begin{array}{l}\text { Densovirus from Acheta domesticus (AdDNV); } 5.5 \mathrm{~kb} \\
\text { genome, ambisense; splicing in VP; small ITRs }\end{array}$ \\
\hline & $1-d$ & (Densovirus) & $\begin{array}{l}\text { Densovirus from Periplaneta fuliginosa (PfDNV); } 5.5 \mathrm{~kb} \\
\text { genome, ambisense; splicing in VP }\end{array}$ \\
\hline & 2 & (Brevidensovirus) & $\begin{array}{l}\text { Genus was earlier named Contravirus by the ICTV, but } \\
\text { these viruses do not have an ambisense organization, } \\
\text { therefore the genus was renamed Brevidensovirus } \\
\text { (Tijssen and Bergoin, 1995). Almost all densoviruses } \\
\text { tentatively classified in this genus by ICTV belong to } \\
\text { other genera. Large number of densoviruses from mos- } \\
\text { quitoes [e.g., from Aedes aegypti (AaeDNV) and from } \\
\text { Aedes albopictus (AalDNV)]. Monosense genome or- } \\
\text { ganization. Genome } 4 \mathrm{~kb} \text { with unique terminal hairpins }\end{array}$ \\
\hline & 3 & (Iteravirus) & $\begin{array}{l}\text { Densonucleosis viruses from Bombyx mori (BmDNV-1) } \\
\text { and Casphalia extranea (CeDNV). Genome } 5 \mathrm{~kb} \text {; ITRs } \\
\text { of about } 225 \text { nucleotides; genome organization } \\
\text { monosense but we observed that the published organi- } \\
\text { zation is incorrect (Zádori et al., unpublished results) }\end{array}$ \\
\hline
\end{tabular}

There are a large number of unclassified viruses (e.g., those from crab and shrimps) that cannot be classified due to a lack of information about their genome. 
There are only a limited number of striking physicochemical differences among the parvoviruses. All parvoviruses have a genome of $4-6 \mathrm{~kb}$ with large palindromic sequences (hairpins) at both ends. The complete virion has a buoyant density of about $1.40 \mathrm{~g} / \mathrm{ml}$ and all have 60 protein subunits. These subunits each consist of one protein from a nested set (co-C-terminal, but different Ntermini) of a few (up to 4) proteins with molecular masses between 40 and $90 \mathrm{kDa}$. The virion is also resistant to various enzymes (proteases, nucleases) and adverse environmental conditions (stable in a wide $\mathrm{pH}$ range, high thermostability). Some parvoviruses encapsidate only the '-' strand (complementary to the mRNA) whereas others encapsidate, into separate virions, also the ' + ' strand (up to $50 \%$, depending on virus and cell). Some viruses, for which both strands are coding, encapsidate both strands equally into separate particles.

\section{Parvovirus infection and replication}

It has been shown for several parvoviruses that nonproductive infections are often due to intracellular factors. These viruses enter both permissive and non-permissive cells, apparently because they often use ubiquitous receptors, but continue only in the permissive cells with a productive infection. This resembles infection by AAVs in the absence of adenovirus. Although integration for autonomous parvoviruses in the absence of replication has not received much attention, densoviruses have been shown to be able to do so (e.g., when an essential gene is knocked out; Bossin, 1998). Minute virus of mice (MVM) directed integration into episomes has also been observed in an experimental system (Corsini et al., 1997). Intracellular factors may thus be important for tropism and interact with the allotropic determinant on the capsid.

The parvoviral genome contains two sets of genes, the nonstructural (NS) or Rep genes and the viral protein (VP) or Cap genes. The NS proteins are central in the replication and expression of the viral genome, while the VP proteins form the capsid to protect the viral genome and play roles in assembly, viral maturation and infection. These VPs are, therefore, far from static proteins and our recent finding of an enzyme activity in the capsid attests to that fact.

Parvoviruses have only a few (2-3) NS proteins to mobilize cellular functions for replication and expression of the viral genome and to execute functions not available in the cell. Sometimes several variants of the NS proteins are generated by minute variations. The largest NS protein, NS-1, has multiple activities, such as ATPase/helicase, nuclear targeting, homo- and hetero-dimerization, transcription-activation domains, DNA-binding and site-specific nickase, and is involved in transcription and replication regulation (reviewed by Vanacker and Rommelaere, 1995). NS-2-negative mutants of MVM grow poorly in mice due 
TIJSSEN

to inefficient translation of viral mRNAs (Brownstein et al., 1992; Naeger et al., 1993). NS-2 has also been reported to be required for the formation of capsids (Cotmore et al., 1997). However, NS-2-knock-out mutants of canine parvovirus (CPV) replicate in dogs and appeared to assemble their capsids in the same way as wild-type virus (Wang et al., 1998). Brockhaus et al. (1996) observed that NS-2 associates with 14-3-3 protein family members that are involved in signal transduction and/or cell cycle regulation pathways.

The role of NS-1 in oncolysis is quite interesting. Cells, even if they proliferate actively, become more sensitive to parvovirus infection upon oncogenic transformation (Rommelaere and Cornelis, 1991). The function inactivation of the tumour suppressor gene product $\mathrm{p} 53$ was found to be paralleled with a sensitization of rat cells with H-1 parvovirus (Telerman et al., 1993). AAV Rep products can also suppress transformation without killing the cells (Schlehofer, 1994).

The linear parvovirus genome has palindromic terminal sequences that can fold into hairpin duplexes (often Y- or T-shaped structure) that serve as primers for replication by host cell DNA polymerase $\delta$ (Cossons et al., 1996) and contain most of the cis-acting elements, both for replication and transcription (Cotmore and Tattersall, 1995). These telomers can vary in size from about 100 to over 500 nucleotides for the different parvoviruses. Some of the parvoviruses (Parvovirus and Brevidensovirus genera) have unique sequences at each end whereas others have inverted terminal repeats (ITRs). The viral DNA is replicated through double-stranded concatemeric intermediates by a unidirectional, quasi-circular rolling-hairpin mechanism (Tattersall and Ward, 1976). The hairpin transfer in the cross-linked ends of the duplex molecules results in a flip/flop sequence inversion (flip is the reverse complementary sequence of flop) and the creation of new replication origins by a terminal resolution reaction (Snyder et al., 1990). For parvoviruses with unique ends, there is a bias toward right-end initiation (Willwand et al., 1998). In addition to nuclear components, NS-1, a pleiotropic viral phosphoprotein of about $80 \mathrm{kDa}$, is critical in this event, by virtue of its site-specific nickase activity (Nuesch et al., 1995), but also for the generation of dimer duplex intermediates. The replicative functions of NS-1 are regulated in turn by phosphorylation through protein kinase $C$ (Nuesch et al., 1998). The left-end origin of some parvoviruses (MVM, PPV) occurs only in the flip orientation and a junction resolution model has been proposed (Liu et al., 1994; Cotmore and Tattersall, 1995). However, many of its elements remain speculative.

The strategies of expression may differ significantly among the various genera. For instance, they may differ with respect to the number of promoters in the genome (1-3), transactivation of promoters, absence or presence of (alternative) splicing, and the use of translational frameshifts. The compact genomes of parvoviruses, except for the largest densoviruses, increase their coding capacity from overlapping reading frames by using alternative splicing. Both elements in introns 
and exons govern these processes in a cis-acting fashion (Pintel et al., 1995). Regulated splicing is also one of the critical factors that control the steady-state levels of the alternative transcripts. In particular, a bipartite enhancer consisting of CA- and purine-rich elements in the NS-2 specific exon mediate proper levels of this exon into the mRNA (Gersappe and Pintel, 1999). Although parvovirus alternative splicing does not seem to be regulated in a temporal fashion during infection, it may be regulated in a cell-type specific way and so have an impact on the tropism of parvoviruses. Liu et al. (1992) observed a block in full-length transcript maturation in cells non-permissive for B19. Also, COS cells, which do not fully support B19, demonstrated a variability in splice boundaries of exon 2 of the 500/600 nucleotide class of RNA when compared to B19-infected human leukemic cells (St Amand et al., 1991).

The necessity for additional chaperone proteins in the formation of empty capsids is not clear and any role of the allotropic determinant at this level remains to be established. Encapsidation of single-stranded DNA requires ongoing DNA synthesis. Defective genomes always contain sequences from the 5 '-end of the '-' strand which could, therefore, contain the cis-acting packaging signal (Faust and Ward, 1979). Since NS-1 proteins are attached to the 5'-ends of all newly synthesized single-stranded DNA (and remain so during packaging; Cotmore and Tattersall, 1989), they may have a role in this process.

\section{Three-dimensional structure of parvoviruses}

The 3D structures of several vertebrate parvoviruses (CPV, FPV, MVM) and, recently, of an invertebrate parvovirus ( $G m D N V)$ have been solved to nearatomic resolution using X-ray crystallography (Simpson et al., 1998). All have a common structure arranged with $\mathrm{T}=1$ icosahedral symmetry with each subunit having the same eight-stranded anti-parallel $\beta$-barrel motif (Fig. 1). This motif contains less than $1 / 3$ of the protein mass of each subunit, is mostly below the capsid surface and consists of two $\beta$-sheets ( $\beta$-strands BIDG and CHEF, respectively). Loops (large insertions between these $\beta$-strands) form much of the capsid surface and interactions with neighbouring subunits. There are three major insertions: between $\beta$-strands B and C (loop 1), between $\beta$-strands E and F (loop 2) and between $\beta$-strands $\mathrm{H}$ and $\mathrm{G}$ (loop 3 for invertebrate parvovirus and loops 3 and 4 for vertebrate parvoviruses). Loops 1 and 2 are centrally located in the asymmetric unit and loops 3 or $3+4$ are found around the 3 -fold axis. In the case of CPV, FPV and MVM, loops 3 and 4 form a $22 \AA$-long spike, whereas for GmDNV, where loop 4 is absent, no spike but a $\beta$-annulus is found at the 3 -fold axis. Although the structure of the human parvovirus, B19, has not been solved to near-atomic resolution, it is clear (Agbandje et al., 1994) that it also lacks the 3-fold spike. 

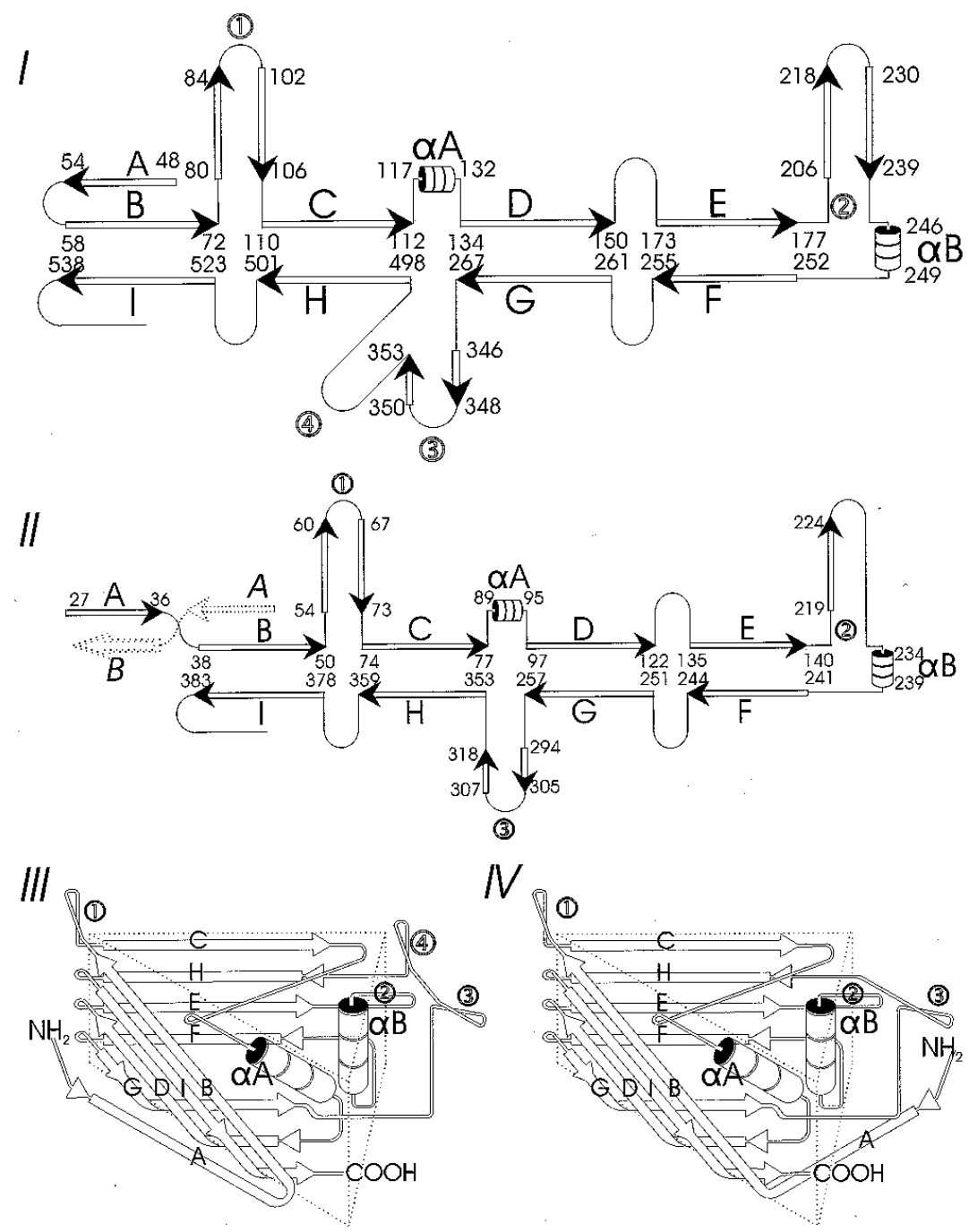

Fig. 1. Diagram of secondary structures of vertebrate and invertebrate parvoviruses. The $\beta$-strands are represented by arrows and the $\alpha$-helices by cylinders. These drawings are not to scale. The loops that interconnect the $\beta$-strands are much longer than the $\alpha$ - and $\beta$-structures and form most of the surface and monomer connecting proteins. The backbone of the capsid structure is the protein sequence in VP2 (for CPV, MVM, PPV) or VP4 (for $G m D N V$ ). Secondary structures are represented in diagrams I (canine parvovirus with numbering of VP2 amino acids) and II ( $G m D N V$ with numbering of VP4 amino acids). The most striking differences are ( $i$ ) the length of the loops; (ii) the presence or absence of loop 4; and, (iii), the orientation of $\beta$-strand A. The $\beta$-barrels (III, IV) are also highly schematic and show how the BIDG and CHEF $\beta$-sheets interact and are quite similar for the vertebrate (III) and invertebrate parvovirus (IV). These triangular subunits correspond to the isometric units represented in Fig. 2. The $\beta$-barrel in GmDNV must be rotated by $7.5^{\circ}$ in order to superimpose the corresponding one in CPV 
Other differences between the vertebrate and invertebrate parvoviruses include the orientation of the $\beta$-strand A which folds back to its own fivefold axis in the case of vertebrate parvoviruses, but interacts with the neighbouring subunit (via the twofold axis) in the case of $G m \mathrm{DNV}$, an invertebrate parvovirus. Also the loops of $G m \mathrm{DNV}$ capsid protein are much shorter, as the mass of the structural proteins of this and related viruses is about $35 \%$ less than that of the vertebrate parvoviruses, and the surface of the capsid is generally much smoother (Fig. 2). It is not clear whether this is a result of the differences in the immune systems in vertebrates and invertebrates (who do not produce immunoglobulins). According to the canyon hypothesis, postulated by Rossmann (1989), vertebrate viruses would have evolved structures to protect crucial sites from antibody recognition (i.e., critical sites would be at the bottom of canyons and inaccessible to antibodies).

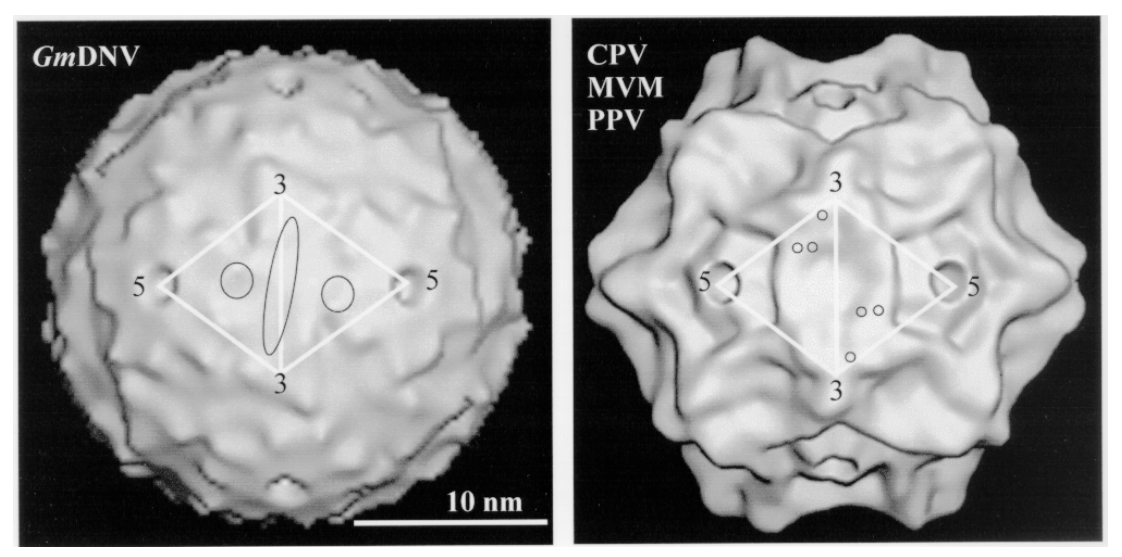

Fig. 2. Computer-generated reconstructions of invertebrate ( $G m \mathrm{DNV})$ and vertebrate (CPV) parvoviruses viewed along the twofold axis. Two isometric units (triangles defined by 3 -fold and 5 -fold axis of symmetry; 60 per particle) are represented on each particle with the positions of the allotropic determinants. The circles in $G m \mathrm{DNV}$ correspond to loop 2 sequences, whereas the circles in the vertebrate parvovirus indicate amino acid differences in the allotropic determinant of PPV

Sequence comparisons demonstrate that $\beta$-strand sequences are more conserved than those of the loops and, as a consequence, the greatest variation occurs at the capsid surface. Closely related viruses (CPV vs. FPV or among biologically different strains of PPV) with very few amino acid differences show that these are located in the loops and often at the surface of the capsid. For $G m \mathrm{DNV}$, most differences are located at the surface and almost exclusively in two domains (not necessarily from the same loop). Since biological differences among these viruses must be accounted for by genetic differences, it was postulated that these few structural differences in the capsid are responsible. 


\section{Allotropic determinants of parvoviruses and their function}

Allotropic determinants have been determined for a few parvoviruses and were found in the capsid (Gardiner and Tattersall, 1988; Chang et al., 1992; Bergeron et al., 1996). Generally, infectious clones (plasmids containing the complete viral genome) were generated that, upon transfection of cells, would excise the viral genome through replication and thus generate virus. This allows the convenient construction of chimeric viruses or mutants and their production in bacteria in order to establish, subsequently, the phenotype of the resultant virus after transfection into host cells. A second prerequisite is the availability of a practical system that allows the recognition of the different phenotypes. For example, in the case of PPV it is known that Kresse and NADL-2 strains have a different phenotype in pigs. NADL-2 virus is hardly pathogenic (low viraemia) whereas the Kresse strain of PPV is highly pathogenic, even to immunocompetent fetuses. It is difficult and costly to find enough PPV-free pigs to study the biological differences between these two virus strains and in vitro systems are needed. However, at this level these virus strains usually do not show recognizable differences and it required a significant effort to find cells that would distinguish these PPV strains. The primary cells (bovine testis) that were able to do so (Bergeron et al., 1996) were then immortalized with SV40 T-antigen and the clones obtained (Laakel et al., unpublished results) demonstrated different phenotypes to these PPV strains (susceptibility vs. non-susceptibility, absence or presence of cytopathic effect in infected cells).

An alternative approach to elucidate parvovirus host range determinants is to pseudotype a genome of a given parvovirus with capsids of closely-related viruses and to study the resultant tropism (Spitzer et al., 1996). It was shown with these transducing particles that the LuIII genome in MVM or H-1 capsids has the tropism of the capsids, not that of the genome.

Only very few genomic differences exist among the strains of PPV (Tijssen et al., 1995; Bergeron et al., 1996) that can be segregated into four, phenotypically distinct, groups. Chimeric constructs generated by exchanging restriction fragments among the infectious clones of these strains pinpointed three amino acid changes in the external loop (3/4) of the capsid protein. In particular one of these (S436P) is prominent as it is located on the top of the threefold spike (Fig. 2). The VP2 differences in yet another strain (P2; Vasudevacharya and Compans, 1992) map structurally closely to the differences we observed between the non-pathogenic NADL-2 and Kresse strains (Bergeron et al., 1996). Oraveerakul et al. (1992) demonstrated that PPV strains may enter nonpermissive cells but that they are restricted at some unknown level.

The sudden emergence of CPV in the late 'seventies and its close relationship to FPV suggested that CPV may have arisen as an FPV variant. Se- 
quence analysis of early CPV strains do not support this hypothesis (Truyen et al., 1998). The initial CPV-2 strains had a strict canine tropism, whereas the strains that replaced these strains in nature in the early 'eighties, i.e. CPV-2a and $2 \mathrm{~b}$, had acquired the ability to infect cats as well $(10 \%$ of parvovirus isolates from feline diagnostic samples is in fact CPV) (Truyen et al., 1996). CPV VP2 residue changes $\mathrm{K} 93 \mathrm{~N}, \mathrm{~A} 103 \mathrm{~V}$, and $\mathrm{D} 323 \mathrm{~N}$ determined that replication could occur in both dogs and canine cells (Chang et al., 1992; Horiuchi et al., 1994). Chimera between FPV and CPV demonstrated that two regions of the genome are important (VP2 residues K80R, N564S, or A568G). These residues are close together in the 3D-structure of the capsid on the top edge of the twofold dimplelike depression in a region where loops 1, 3, and 4 of three different monomers interact. Additional changes in this area may revert the CPV strain to a feline host range (Truyen and Parrish, 1995). A single mutation in VP2, A300D, causes a loss in canine host range (Llamas-Saiz et al., 1996). This D300 forms a salt bridge with R81 inducing also local changes within the antigenic site. In addition, the loop between residues 359 and 374 (in loop 4) adopts a structure similar to that of FPV. Horiuchi et al. (1992) observed that the stage in FPV virus replication cycle at which the host specificity of this subgroup is regulated in canine cells is found after cell entry of the virus.

It was observed for MVM that the MVMp strain infects fibroblast cells (A9) and the MVMi strain infects lymphoid cells (EL4 T-lymphocytes). The fibrotropic determinant in MVMp was mapped to residues 317 and 321 and MVMi could become fibrotropic by mutations at these positions (Ball-Goodrich et al., 1991). In contrast, two segments of MVMi (one in the NS and one in the VP region) were required to confer its phenotype to MVMp (Colomar et al., 1998). The NS segment influences apparently the virus-strain-specific differences in the regulation of splicing, whereas the VP segment impacts on the virion structure. Mutations in NS genes that affected the expression of the replicative form of the viral DNA restricted the viral reproduction more in murine cells than in other cells (Naeger et al., 1993). Spalholz and Tattersall (1983) also demonstrated that the strain-specific target cell specificity is mediated by intracellular factors.

MVM and PPV resemble each other in several aspects: (i) the nonpathogenic PPV-NADL-2 and MVMp strains are much less viraemic in vivo than the pathogenic strains; (ii) the fetal infection by PPV finds its equivalent in the generalized infection for MVM of hematopoietic cells and the capillary endothelium; and (iii) the position and function of the allotropic determinants. Unfortunately, we do not have yet the sequence of the NADL-8 strain (only pathogenic to non-immunocompetent fetuses), which has been shown to have a very different tropism towards the various organs when compared to the pathogenic Kresse strain (Oraveerakul et al., 1993). 
There is a considerable variability among the different strains of ADV, particularly in a hypervariable region of about 25 nucleotides in the capsid gene. Some ADV strains grow only in the animal (e.g. ADV-Utah) whereas others grow in tissue culture (e.g. the nonpathogenic ADV-G). Chimeric constructs of an infectious clone of ADV-G with wild-type sequences indicated that sequences within 55-65 m.u. of ADV-Utah inhibited replication in vitro (Bloom et al., 1993). Construction of pathogenic molecular clones of ADV that replicate both in vivo and in vitro revealed two supplementary regions, at 65-69 and 7388 m.u., that, in tandem, can abolish growth in vitro (Bloom et al., 1998). A novel ADV strain (ADV-TR) that is closely related to ADV-G, but is pathogenic for Aleutian mink, was found to be able to infect raccoons and could be responsible for transmission of ADV infections (Oie et al., 1996). Twelve amino acids in the VP region were identified that could be at the basis of this phenotype.

The identification of the allotropic determinant of $G m \mathrm{DNV}$ is also underway. This virus group offers the advantage that the host animals can be manipulated easily. Infectious clones have been generated and exchange of restriction fragments have been shown that two regions on the capsid are implicated (Fig. 2), one is around loop 2 in the middle of the isometric subunit and one straddles loop 3 and is continuous in two subunits along the 3 -fold to 3 -fold axis. Further investigations should allow us to reduce the number of amino acids that are essential for the species-specific tropism.

\section{Conclusion}

The various parvoviruses for which tropism has been studied all show that minimal changes in the sequence of the structural protein can determine cell tropism or host range. The location of these critical amino acids, usually in loops 3 and 4 and at the C-terminus of the capsid protein, are at or near the capsid surface, suggesting strongly that they interact with a cellular molecule. Ample evidence indicates that this cellular counterpart is not the receptor. Two possibilities exist: the cellular factor enables the virus infection in permissive cells or inhibits virus infections in non-permissive cells. Although the decapsidation stage seems to be a logical candidate, the limited replication and expression may indicate that later steps in the viral cycle are critical.

The sudden and devastating emergence of MEV and CPV in the last 50 years demonstrates the importance of parvovirus tropism. Whether they arose by a few mutations from FPV or have another origin is academic. Despite its importance and considerable efforts, we know still precious little about the mechanisms of tropism changes of this virus group. 


\section{Acknowledgements}

I wish to acknowledge the help from my collaborators at Purdue (M. Rossmann and A. Simpson), Cornell (C. Parrish), VIDO (L. Babiuk) and University of Montpellier/INRA (M. Bergoin, F.-X. Jousset) and from everybody in my laboratory working on this project, especially the post-doctoral fellows (M. Laakel, Y. Li, and Z. Zádori), students (M. David, S. Forest, S. Gariépy, B. Hébert, M.-C. Lacoste), research assistant (M. Letarte) and technical staff (J. Reid, L. Forget, L. Paris-Nadon). Furthermore, I want to express my gratitude to the Natural Science and Engineering Research Council of Canada for their financial support.

\section{References}

Agbandje, M., Kajigaya, S., McKenna, R., Young, N. S. and Rossmann, M. G. (1994): The structure of human parvovirus B19 at $8 \AA$ resolution. Virology 203, 106-115.

Ball-Goodrich, L. J., Moir, R. D. and Tattersall, P. (1991): Parvoviral target cell specificity acquisition of fibrotropism by a mutant of the lymphotropic strain of minute virus mice involves multiple amino acid substitutions within the capsid. Virology 184, 175-186.

Bergeron, J., Hébert, B. and Tijssen, P. (1996): Genome organization of the Kresse strain of porcine parvovirus. Identification of the allotropic determinant and its comparison with those of NADL-2 and field strains. J. Virol. 70, 2508-2515.

Berns, K. I. (1996): Parvoviridae: the viruses and their replication. In: Fields, B. N., Knipe, D. M. and Howley, P. M. (eds) Fundamental Virology, $3^{\text {rd }}$ edition. Lippencott-Raven Publishers, Philadelphia. pp. 1017-1041.

Berns, K. I., Bergoin, M., Bloom, M., Lederman, M., Muzyczka, N., Siegl, G., Tal, J. and Tattersall, P. (1995): Parvoviridae. VIth report of International Committee on Taxonomy of Viruses. Arch. Virol. Suppl. 10, 169-178.

Bloom, M. E., Berry, B. D. Wei, W., Perryman, S. and Wolfinbarger, J. B. (1993): Characterization of chimeric full-length molecular clones of Aleutian mink disease parvovirus (ADV): identification of a determinant governing replication in cell culture. J. Virol. 67, 5976-5988.

Bloom, M. E., Fox, J. M., Berry, B. D., Oie, K. L. and Wolfinbarger, J. B. (1998): Construction of pathogenic molecular clones of Aleutian mink disease parvovirus that replicate both in vivo and in vitro. Virology 251, 288-296.

Bossin, H. (1998): Development of stable expression vectors derived from $J_{c D N V}$ : application to constitutive expression of heterologous proteins in Lepidopteran cell lines and as a marker during Drosophila development [in French]. Ph. D. Thesis, University of Montpellier II, France. 226 pp.

Brockhaus, K., Plaza, S., Pintel, D. J., Rommelaere, J. and Salomé, N. (1996): Nonstructural proteins NS2 of minute virus of mice associate in vivo with 14-3-3 protein family members. J. Virol. 70, 7527-7534.

Brownstein, D. G., Smith, A. L., Johnson, E. A., Pintel, D. J., Naeger, L. K. and Tattersall, P. (1992): The pathogenesis of infection with minute virus of mice depends on expression of the small nonstructural protein NS2 and on the genotype of the allotropic determinants VP1 and VP2. J. Virol. 66, 3118-3124.

Chang, S.-F., Sgro, J.-Y. and Parrish, C. R. (1992): Multiple amino acids in the capsid structure of canine parvovirus coordinately determine the canine host range and specific antigenic and hemagglutinating properties. J. Virol. 66, 6858-6867. 
Colomar, M. C., Hirt, B. and Beard, P. (1998): Two segments in the genome of the immunosuppressive minute virus of mice determine the host-cell specificity, control viral DNA replication and affect viral RNA metabolism. J. Gen. Virol. 79, 581-586.

Corsini, J., Tal, J. and Winocour, E. (1997): Directed integration of minute virus of mice DNA into episomes. J. Virol. 71, 9008-9015.

Cossons, N., Faust, E. A. and Zannis-Hadjopoulos, M. (1996): DNA polymerase delta-dependent formation of a hairpin structure at the 5 ' terminal palindrome of the minute virus of mice genome. Virology 216, 258-264.

Cotmore, S. F. and Tattersall, P. (1989): A genome-linked copy of the NS-1 polypeptide is located on the outside of infectious parvovirus particles. J. Virol. 63, 3902-3911.

Cotmore, S. F. and Tattersall, P. (1995): DNA replication in the autonomous parvoviruses. Sem. Virol. 6, 271-281.

Cotmore, S. F., D'Abramo, A. M., Jr., Carbonell, L. F., Bratton, J. and Tattersall, P. (1997): The NS2 polypeptide of parvovirus MVM is required for capsid assembly in murine cells. Virology 231, 267-280.

Faust, E. A. and Ward, D. C. (1979): Incomplete genomes of the parvovirus minute virus of mice: selective conservation of genomic termini. J. Virol. 32, 276-292.

Gardiner, E. M. and Tattersall, P. (1988): Mapping of the fibrotropic and lymphotropic host range determinants of the parvovirus minute virus of mice. J. Virol. 62, 2605-2613.

Gersappe, A. and Pintel, D. J. (1999): CA- and purine-rich elements form a novel bipartite exon enhancer which governs inclusion of the minute virus of mice NS2-specific exon in both singly and doubly spliced mRNAs. Mol. Cell. Biol. 19, 364-375.

Gibbs, A. I. and Harrison, B. O. (1968): Realistic approach to virus classification and nomenclature. Nature 218, 927-929.

Horiuchi, M., Ishiguro, N., Goto, H. and Shinagawa, M. (1992): Characterization of the stage(s) in the virus replication cycle at which the host-cell specificity of the feline parvovirus subgroup is regulated in canine cells. Virology 189, 600-608.

Horiuchi, M., Goto, H., Ishiguro, N. and Shinagawa, M. (1994): Mapping of the determinants of the host range for canine cells in the genome of canine parvovirus using canine parvovirus/mink enteritis chimeric viruses. J. Gen. Virol. 75, 1319-1328.

Kotin, R. M., Linden, R. M. and Berns, K. I. (1992): Characterization of a preferred site on human chromosome 19q for integration of adeno-associated virus DNA by non-homologous recombination. EMBO J. 11, 5071-5078.

Liu, J. M., Green, S. W., Shimada, T. and Young, N. S. (1992): A block in full-length transcript maturation in cells nonpermissive for B19 parvovirus. J. Virol. 66, 4686-4692.

Liu, Q., Yong, C. B. and Astell, C. R. (1994): In vitro resolution of the dimer bridge of the minute virus of mice (MVM) genome supports the modified rolling hairpin model for MVM replication. Virology 201, 251-262.

Llamas-Saiz, A. L., Agbandje-McKenna, M., Parker, J. S., Wahid, A. T., Parrish, C. R. and Rossmann, M. G. (1996): Structural analysis of a mutation in canine parvovirus which controls antigenicity and host range. Virology $225,65-71$.

Lwoff, A. (1967): Principles of classification and nomenclature of viruses. Nature 215, 13-14.

Mayor, H. D., Drake, S., Stahmann, J. and Mumford, D. M. (1976): Antibodies to adenoassociated satellite virus and herpes simplex in sera from cancer patients and normal adults. Am. J. Obstet. Gynecol. 126, 100-104.

Naeger, L. K., Salomé, N. and Pintel, D. J. (1993): NS2 is required for efficient translation of viral mRNA in minute virus of mice-infected murine cells. J. Virol. 67, 1034-1043.

Nuesch, J. P. F., Cotmore, S. F. and Tattersall, P. (1995): Sequence motifs in the replicator protein of parvovirus MVM essential for nicking and covalent attachment to the viral origin: identification of the linking tyrosine. Virology 209, 122-135. 
Nuesch, J. P., Dettwiler, S., Corbau, R. and Rommelaere, J. (1998): Replicative functions of minute virus of mice NS1 protein are regulated in vitro by phosphorylation through protein kinase C. J. Virol. 72, 9966-9977.

Oie, K. L., Durrant, G., Wolfinbarger, J. B., Martin, D., Costello, F., Perryman, S., Hogan, D., Hadlow, W. J. and Bloom, M. E. (1996): The relationship between capsid protein (VP2) sequence and pathogenicity of Aleutian mink disease parvovirus (ADV): a possible role for raccoons in the transmission of ADV infections. J. Virol. 70, 852-861.

Oraveerakul, K., Choi, C. S. and Molitor, T. W. (1992): Restriction of porcine parvovirus replication in nonpermissive cells. J. Virol. 66, 715-722.

Oraveerakul, K., Choi, C. S. and Molitor, T. W. (1993): Tissue tropisms of porcine parvovirus in swine. Arch. Virol. 130, 377-389.

Pintel, D. J., Gersappe, A., Haut, D. and Pearson, P. (1995): Determinants that govern alternative splicing of parvovirus pre-mRNAs. Sem. Virol. 6, 283-290.

Rommelaere, J. and Cornelis, J. J. (1991): Antineoplastic activity of parvoviruses. J. Virol. Meth. 33, 233-251.

Rossmann, M. G. (1989): The canyon hypothesis. Hiding the host cell receptor attachment site on a viral surface from immune surveillance. J. Biol. Chem. 264, 14587-14590.

Schlehofer, J. R. (1994): The tumor suppressive properties of adeno-associated viruses. Mutation Res. 305, 303-313.

Siegl, G. (1976): The parvoviruses. Virol. Monogr. 15, 1-109.

Simpson, A. A., Chipman, P. R., Baker, T. S., Tijssen, P. and Rossmann, M. G. (1998): The structure of an insect parvovirus (Galleria mellonella densovirus) at $3.7 \AA$ resolution. Structure 6, 1355-1367.

Snyder, R. O., Samulski, R. J. and Muzyczka, N. (1990): In vitro resolution of covalently joined AAV chromosome ends. Cell 60, 105-113.

Spalholz, B. A. and Tattersall, P. (1983): Interaction of minute virus of mice with differentiated cells: strain-dependent target cell specificity is mediated by intracellular factors. J. Virol. 46, 937-943.

Spitzer, A. L., Maxwell, F., Corsini, J. and Maxwell, I. H. (1996): Species specificity for transduction of cultured cells by a recombinant LuIII rodent parvovirus genome encapsidated by canine parvovirus or feline panleukopenia virus. J. Gen. Virol. 77, 1787-1792.

St Amand, J., Beard, C., Humphries, K. and Astell, C. R. (1991): Analysis of splice junctions and in vitro and in vivo translation potential of the small, abundant B19 parvovirus RNAs. Virology 183, 133-142.

Tattersall, P. and Ward, D. C. (1976): Rolling hairpin model for replication for replication of parvovirus and linear chromosomal DNA. Nature 263, 106-109.

Telerman, A., Tuynder, M., Dupressoir, T., Robaye, B., Sigaux, F., Shaulian, E., Oren, M., Rommelaere, J. and Amson, R. (1993): A model for tumor suppression using parvovirus H-1. Proc. Natl. Acad. Sci. USA 90, 8702-8706.

Tijssen, P. (Editor) (1990): Handbook of Parvoviruses vol. I (309 pp) and vol. II (312 pp). CRC Press, Boca Raton, Florida.

Tijssen, P. and Bergoin, M. (1995): Densonucleosis viruses constitute an increasingly diversified family among parvoviruses. Sem. Virol. 6, 347-355.

Tijssen, P., Bergeron, J., Dubuc, R. and Hébert, B. (1995): Minor genetic changes among porcine parvovirus groups are responsible for major distinguishing properties. Sem. Virol. 6, 319-328.

Tijssen, P., Laakel, M., Zádori, Z. and Hébert, B. (1999): Parvoviruses: rodents, pigs, cattle and waterfowl. In: Webster, R. G. and Granoff, A. (eds) Encyclopedia in Virology. $2^{\text {nd }}$ edition. Academic Press, San Diego, CA (in press).

Truyen, U. and Parrish, C. R. (1995): The evolution and control of parvovirus host ranges. Sem. Virol. 6, 311-317. 
Truyen, U., Evermann, J. F., Vieler, E. and Parrish, C. R. (1996): Evolution of canine parvovirus involved loss and gain of feline host range. Virology 215, 186-189.

Truyen, U., Geissler, K., Parrish, C. R., Hermanns, W. and Siegl, G. (1998): No evidence for a role of modified live virus vaccines in the emergence of canine parvovirus. J. Gen. Virol. 79, $1153-1158$

Vasudevacharya, J. and Compans, R. W. (1992): The NS and capsid genes determine the host range of porcine parvovirus. Virology 187, 515-524.

Vanacker, J.-M. and Rommelaere, J. (1995): Non-structural proteins of autonomous parvovirus: from cellular effects to molecular mechanisms. Sem. Virol. 6, 291-297.

Wang, D., Yuan, W., Davis, I. and Parrish, C. R. (1998): Nonstructural protein-2 and the replication of canine parvovirus. Virology 240, 273-281.

Willwand, K., Mumtsidu, E., Kuntz-Simon, G. and Rommelaere, J. (1998): Initiation of DNA replication at palindromic telomeres is mediated by a duplex-to-hairpin transition induced by the minute virus of mice nonstructural protein NS1. J. Biol. Chem. 273, 1165-1174.

Zádori, Z., Stefancsik, R., Rauch, T. and Kisary, J. (1995): Analysis of the complete sequences of goose and muscovy duck parvoviruses indicates common ancestral origin with adenoassociated virus 2 . Virology $212,562-573$. 
0236-6290/99/\$ 5.00 @ 1999 Akadémiai Kiadó, Budapest 